\title{
Beam guiding system geometric arrangement in the target area of high-power laser drivers
}

\author{
Lei Ren, Dongfeng Zhao, and Jianqiang Zhu \\ National Laboratory on High Power Laser and Physics, Shanghai Institute of Optics and Fine Mechanics, Chinese Academy of Science, \\ Shanghai 201800, China \\ (Received 7 December 2014; revised 18 January 2015; accepted 6 February 2015)
}

\begin{abstract}
With the increasing number of laser beams, the main difficulty in arranging beam guiding systems (BGSs) involves determining the corresponding relationships between the output and input ports to realize the identified light path length of all beams. Given the basic constraints of geometric arrangement, a BGS model is established, and a base-line algorithm is proposed to address the difficulty mentioned above. Boundary conditions of target area and target chamber are discussed to increase the number of laser beams, and a maximum value exists for a specific target area. Finally, the compatibility of a cylindrical hohlraum target chamber with a spherical hohlraum is analyzed, and a moveable final optics assembly is proposed to execute the switch between the two different targets.
\end{abstract}

Keywords: beam guiding system; laser drivers; spherical hohlraum; target areas

\section{Introduction}

A high-power laser driver is a massive facility aimed at inertial confinement fusion (ICF) ${ }^{[1,2]}$. It is a promising way to realize ICF if the output power is about 1-2 MJ at $351 \mathrm{~nm}$ with a pulse width of $1-5 \mathrm{~ns}^{[3]}$. Based on experiments ${ }^{[4]}$ carried out at the National Ignition Facility (NIF) ${ }^{[5]}$ with laser powers reaching $520 \mathrm{TW}$ and laser energies of up to $1.9 \mathrm{MJ}$, although the ignition failed, it was suggested that the achievement of higher laser powers and energies to increase the $\mathrm{x}$-ray drive and the margin for ICF has become possible. Besides trying to improve the output power per laser beam, the other approach to obtain higher energy is to increase the number of beam lines. Increasing the number of laser beams is considerably safer considering that laser damage to optical components requires less attention. In addition, the risk for regular operation is lower, given that the single-beam energy will decrease because of the fixed total operation energy. Furthermore, better confinement symmetry can be ensured for the indirect and direct methods if many more laser beams are used to shoot the target.

The earliest study of a multi-beam laser driver beam guiding system (BGS) can be traced back to Shiva ${ }^{[6]}$. A 20-laserbeam facility with a total output energy fluence of $10 \mathrm{~kJ}$ was invented at the Lawrence Livermore National Laboratory (LLNL) in the 1970s. Shiva's BGS is relatively simple

Correspondence to: J. Zhu, No. 390, Qinghe Rd., Jiading, Shanghai 201800, China. Email: jqzhu@mail.shcnc.ac.cn and involves only two mirrors. After amplification, the laser beams are guided by these two mirrors, which redirect their angles to meet the physical requirements and shoot the target. In the 1980s, almost simultaneously, when LLNL was upgrading the Nova facility ${ }^{[3]}$ and the French Commissariat à l'Energie Atomique (CEA) was upgrading the Phébus facility ${ }^{[7]}$, both research bodies considered increasing their laser beam numbers to 288 to obtain a laser energy that exceeded 1 MJ laser energy. Two BGS concept design configurations were proposed, namely, crosswise orientation and in-line orientation ${ }^{[8]}$. Given the disadvantages of inline orientation, the BGS configurations of high-power laser drivers that have subsequently been built have all been based on crosswise orientation, but with varying shapes. For example, NIF possesses 192 laser beams, and every quad consists of four beams, with 48 quads in total. In the switchyard of NIF, every quad is guided by four or five mirrors into the target chamber. The BGS and target chamber make the shape of the character ' $U$ '[9, 10]. The Laser Mégajoule (LMJ) Project ${ }^{[11]}$ built by CEA possesses 240 laser beams, and one quad also includes four beams. Its BGS is composed of six mirrors and directly inherits the configuration of crosswise orientation, which looks like the character ' $\mathrm{I}$ '[12]. The in-built SG-III ${ }^{[13]}$ in China consists of 60 laser beams, and each line is guided by four mirrors, making the BGS L-shaped ${ }^{[14]}$. The evolution of the BGS configuration discussed above is pictured in Figure 1. 


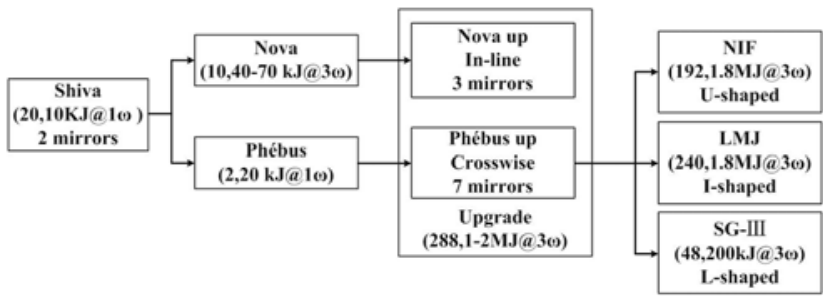

Figure 1. The evolution of the entire BGS configuration.

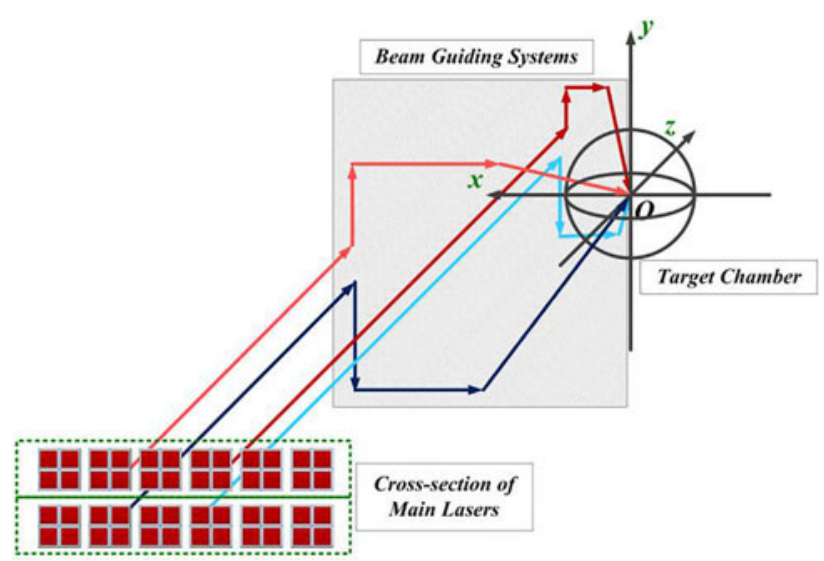

Figure 2. Simplified scheme of a light path from the spatial filter to the target chamber center.

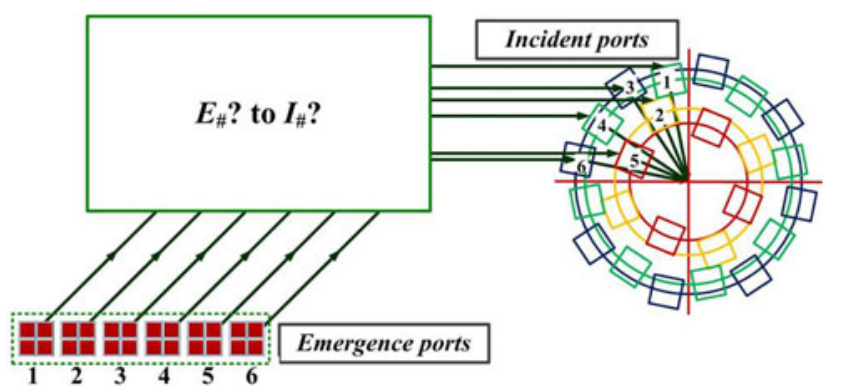

Figure 3. The fundamental problem of BGS arrangement.

\section{Modeling of BGSs}

\subsection{Basic function of BGSS}

In the target area of high-power laser drivers, the process of main laser propagation can be simplified as follows: after exiting from the last spatial filter, the main lasers are guided by several mirrors, redirected to meet the angle requirement of the physics experiments, and then shot through the final optics assembly (FOA) to the center of the target chamber, as shown in Figure 2. Thus, the BGS maps the rectangular arrangement at the laser output to a spherical geometry configuration at the target chamber. In addition, all the laser beams share the same light path length (LPL) when they arrive at the target. Furthermore, all reflections at the mirrors in the BGS are in-plane (either S or P) and the incident light must be $\mathrm{P}$ polarized. Finally, no obscuring or intersection occurs among laser beams when propagating.

\subsection{Fundamental problems of BGS arrangement}

Taking six laser beams in Figure 2 as an example, after being redirected by the BGS, they will propagate to the target through six incident ports in the chamber. As shown in Figure 3, the distribution of incident ports is determined by the physics requirement, and the emergence ports are determined by the laser amplifiers. Therefore, the fundamental problem of BGS arrangement involves determining the correspondence between the emergence and incident ports, namely, the $E_{\#}$ and $I_{\#}$ shown in Figure 3, so that all the laser beams can meet the requirements of the physics experiment.

\subsection{Typical constraints in BGS modeling}

From the viewpoint of laser driver construction, the first constraint is the space of the target area and target chamber. Then, considering the mirror coatings, all incidence angles of each mirror must be less than $45^{\circ}$. Moreover, the number
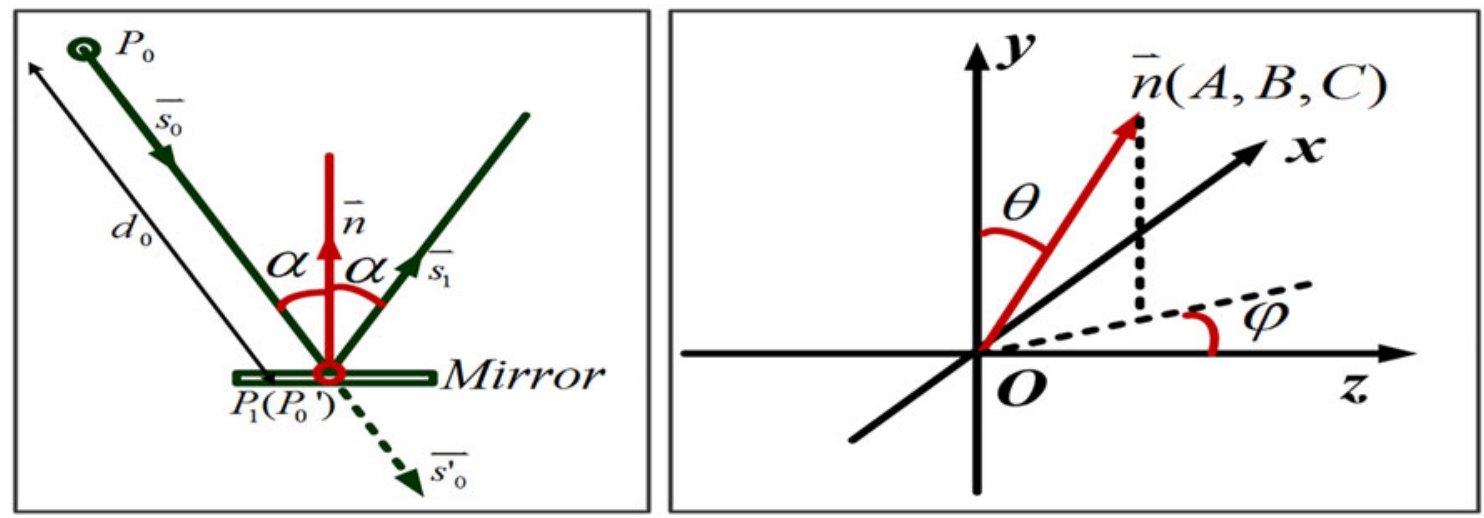

Figure 4. The process of a light propagating a certain distance and being reflected by one mirror. 
and types of mirrors must be as small as possible so that the BGS will be simple and easy to realize. The final constraint is the total BGS configuration, and the following modeling is based on a U-shaped configuration.

\subsection{Multi-beam BGS modeling}

Given that the BGS is constructed from several mirrors, analysis of the process of light reflected by a mirror is crucial. As pictured in Figure 4, a light can be expressed as an emergence point and a direction vector. One light travels along the direction vector $s_{0}\left(a_{0}, b_{0}, c_{0}\right)$ from an emergence point $P_{0}\left(x_{0}, y_{0}, z_{0}\right)$ to a point $P_{0}^{\prime}$ in a mirror, and the length of the light path is $d_{0}$. The propagating matrix can be calculated through Equation (1a), where the vector $s_{0}$ is equal to $s_{0^{\prime}}$. After being reflected by a mirror, whose unit normal vector is $n(A, B, C)$, the emergence point and direction vector of the light become $P_{1}$ and $s_{1}$, respectively. The propagating matrix can be calculated through Equation $(1 b)$, where the emergence point $P_{1}$ is equal to $P_{0}^{\prime}$. By combining the two processes, we obtain the transforming matrix from incident light to emergent light after propagating a certain distance and being reflected by one mirror, as shown in Equation (1c), where $A=$ $\sin \theta \sin \varphi, B=\cos \theta, C=\sin \theta \cos \varphi$,

$$
\begin{aligned}
& \left(\begin{array}{l}
x_{0}^{\prime} \\
y_{0}^{\prime} \\
z_{0}^{\prime} \\
a_{0}^{\prime} \\
b_{0}^{\prime} \\
c_{0}^{\prime}
\end{array}\right)=M_{1}\left(\begin{array}{l}
x_{0} \\
y_{0} \\
z_{0} \\
a_{0} \\
b_{0} \\
c_{0}
\end{array}\right) \\
& =\left(\begin{array}{cccccc}
1 & 0 & 0 & d_{0} & 0 & 0 \\
0 & 1 & 0 & 0 & d_{0} & 0 \\
0 & 0 & 1 & 0 & 0 & d_{0} \\
0 & 0 & 0 & 1 & 0 & 0 \\
0 & 0 & 0 & 0 & 1 & 0 \\
0 & 0 & 0 & 0 & 0 & 1
\end{array}\right)\left(\begin{array}{c}
x_{0} \\
y_{0} \\
z_{0} \\
a_{0} \\
b_{0} \\
c_{0}
\end{array}\right), \\
& \left(\begin{array}{l}
x_{1} \\
y_{1} \\
z_{1} \\
a_{1} \\
b_{1} \\
c_{1}
\end{array}\right)=M_{2}\left(\begin{array}{l}
x_{0}^{\prime} \\
y_{0}^{\prime} \\
z_{0}^{\prime} \\
a_{0}^{\prime} \\
b_{0}^{\prime} \\
c_{0}^{\prime}
\end{array}\right) \\
& =\left(\begin{array}{cccccc}
1 & 0 & 0 & 0 & 0 & 0 \\
0 & 1 & 0 & 0 & 0 & 0 \\
0 & 0 & 1 & 0 & 0 & 0 \\
0 & 0 & 0 & 1-2 A^{2} & -2 B A & -2 C A \\
0 & 0 & 0 & -2 A B & 1-2 B^{2} & -2 C B \\
0 & 0 & 0 & -2 A C & -2 B C & 1-2 C^{2}
\end{array}\right)\left(\begin{array}{c}
x_{0}^{\prime} \\
y_{0}^{\prime} \\
z_{0}^{\prime} \\
a_{0}^{\prime} \\
b_{0}^{\prime} \\
c_{0}^{\prime}
\end{array}\right),
\end{aligned}
$$

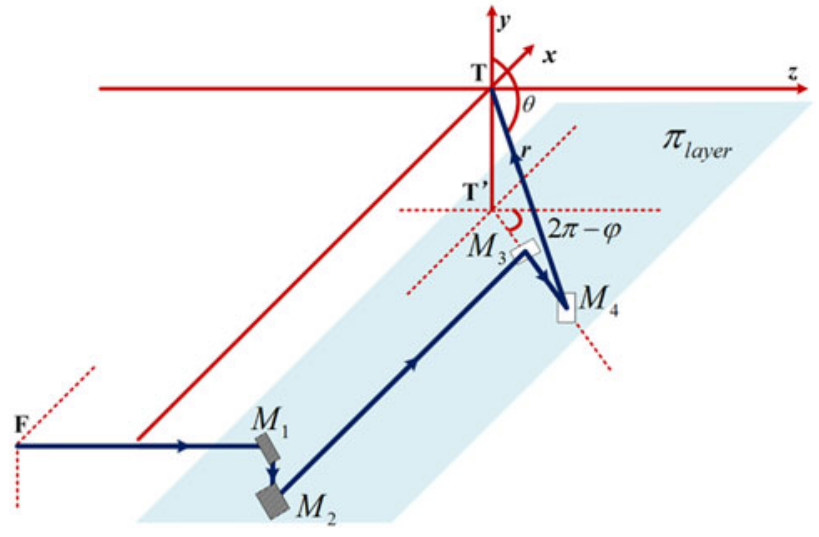

Figure 5. Model of a single light guided by a BGS.

$$
\begin{gathered}
\left(\begin{array}{l}
x_{1} \\
y_{1} \\
z_{1} \\
a_{1} \\
b_{1} \\
c_{1}
\end{array}\right)=T\left(\begin{array}{l}
x_{0} \\
y_{0} \\
z_{0} \\
a_{0} \\
b_{0} \\
c_{0}
\end{array}\right)=M_{2} M_{1}\left(\begin{array}{l}
x_{0} \\
y_{0} \\
z_{0} \\
a_{0} \\
b_{0} \\
c_{0}
\end{array}\right) \\
=\left(\begin{array}{llllcc}
1 & 0 & 0 & d_{0} & 0 & 0 \\
0 & 1 & 0 & 0 & d_{0} & 0 \\
0 & 0 & 1 & 0 & 0 & d_{0} \\
0 & 0 & 0 & 1-2 A^{2} & -2 B A & -2 C A \\
0 & 0 & 0 & -2 A B & 1-2 B^{2} & -2 C B \\
0 & 0 & 0 & -2 A C & -2 B C & 1-2 C^{2}
\end{array}\right)\left(\begin{array}{l}
x_{0} \\
y_{0} \\
z_{0} \\
a_{0} \\
b_{0} \\
c_{0}
\end{array}\right) .
\end{gathered}
$$

Based on Equation (1c), with only two mirrors, one laser beam can be transported from one point to any other point in any direction without any constraints. However, according to the incident angle and polarization requirements and all the constraints discussed above, the configuration of singlelight propagation can be designed in Figure 5. The main lasers emerge from the center point $F$ of the spatial filter and reach the target chamber center $T$ after being reflected by four mirrors $M_{j}(j=1,2,3,4)$. Mirror 1 only changes the light path in the $y$ direction and mirror 2 does so in the $x$ direction. The light path from mirror 3 to mirror 4 is at the same altitude, and the projection of the line $M_{4} T$ in the $X Z$ plane is the line $T^{\prime} M_{3} M_{4}$ to ensure that the incident light is $P$ polarized.

The angle between the incident light and the $y$-axis positive direction is defined as $\theta(0 \leqslant \theta \leqslant \pi)$, the angle between the projection of incident light and the $z$-axis positive direction is $\varphi(0 \leqslant \varphi \leqslant 2 \pi)$ and the counterclockwise direction is positive. Mirrors 2, 3 and 4 are supposed to be in the same plane $\pi_{\text {layer }}$. The clear radius is defined to be the distance between the target chamber center $T$ and the fourth mirror $M_{4}$ and its length is $r$. In order to avoid obscuring and 


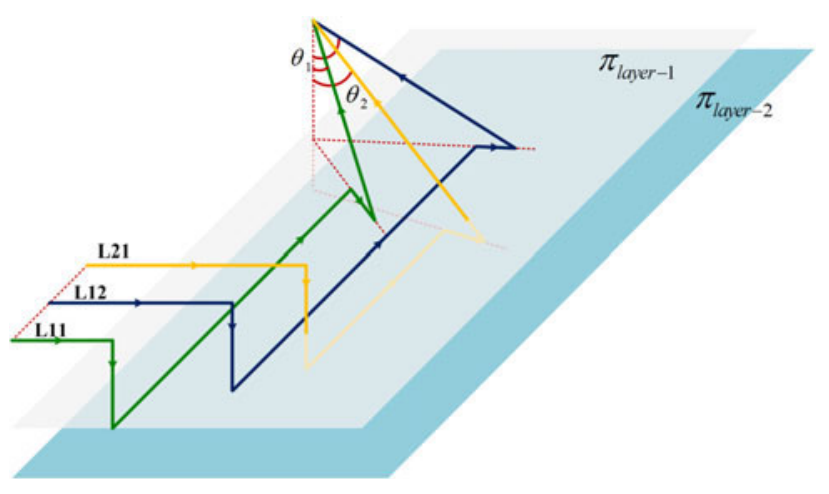

Figure 6. Scheme of different lights guided by a BGS. L11 and L12 possess the same incident angle so they are in plane layer 1. Meanwhile, L21 with a different incident angle is in plane layer 2 .

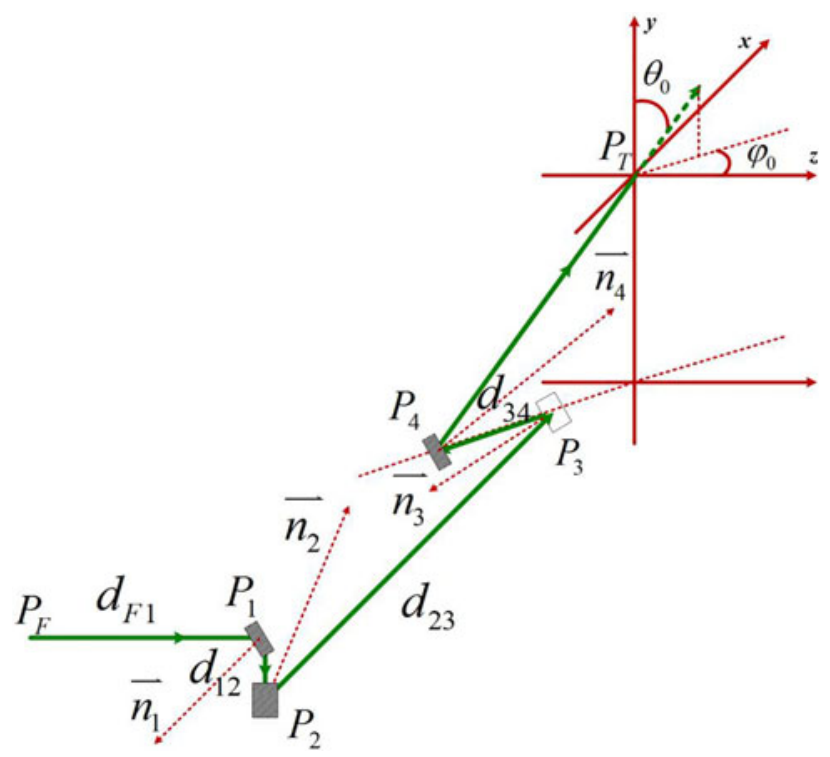

Figure 7. The process of a light propagating a certain distance and being reflected by four mirrors.

intersection among the laser beams when propagating, the clear radii of incident light rays with the same $\theta$ should be equal. In this manner, laser beams with different incident angles will travel in different planes or layers. The model of multiple beams guided by a BGS is plotted in Figure 6, which shows that all lights are arranged in different layers according to their incident angles, and no intersection should occur among the beams in the different layers.

According to Equation (1c) and Figure 5, the propagation process can be pictured in Figure 7. Given the original emergence direction vectors and unit normal vectors of the four mirrors in Equation (2a), the expression of each emergence point and direction vector can be calculated with Equation $(2 b)$. Because $P_{F}, \theta_{0}$ and $\varphi_{0}$ are constant variables that are determined by the laser amplifiers and target chamber, supposing that the clear radius $r$ is fixed, then the position of mirror $4, P_{4}$, and the direction vector $s_{4}$ can be calculated. According to Equation (2c), the distance between two adjacent mirrors and the total LPL can be expressed in Equation $(2 d)$,

$$
\begin{aligned}
& \left\{\begin{array}{l}
a_{f}=0, \quad b_{f}=0, \quad c_{f}=1 \\
A_{1}=0, \quad B_{1}=\frac{-\sqrt{2}}{2}, \quad C_{1}=\frac{-\sqrt{2}}{2} \\
A_{2}=\frac{\sqrt{2}}{2}, \quad B_{2}=\frac{\sqrt{2}}{2}, \quad C_{2}=0 \\
A_{3}=-\sin \left[\frac{\pi}{4}+\frac{\phi_{0}}{2}\right], \quad B_{3}=0, \\
C_{3}=-\cos \left[\frac{\pi}{4}+\frac{\phi_{0}}{2}\right] \\
A_{4}=\sin \left[\frac{\pi}{4}+\frac{\theta_{0}}{2}\right] \sin \left[\phi_{0}\right], \quad B_{4}=\cos \left[\frac{\pi}{4}+\frac{\theta_{0}}{2}\right], \\
C_{4}=\sin \left[\frac{\pi}{4}+\frac{\theta_{0}}{2}\right] \cos \left[\phi_{0}\right]
\end{array}\right. \\
& {\left[\begin{array}{l}
x_{1} \\
y_{1} \\
z_{1} \\
a_{1} \\
b_{1} \\
c_{1}
\end{array}\right]=\left[\begin{array}{c}
x_{f} \\
y_{f} \\
d_{F 1}+z_{f} \\
0 \\
-1 \\
0
\end{array}\right], \quad\left[\begin{array}{c}
x_{2} \\
y_{2} \\
z_{2} \\
a_{2} \\
b_{2} \\
c_{2}
\end{array}\right]=\left[\begin{array}{c}
x_{f} \\
-d_{12}+y_{f} \\
d_{F 1}+z_{f} \\
1 \\
0 \\
0
\end{array}\right],} \\
& {\left[\begin{array}{l}
x_{3} \\
y_{3} \\
z_{3} \\
a_{3} \\
b_{3} \\
c_{3}
\end{array}\right]=\left[\begin{array}{c}
d_{23}+x_{f} \\
-d_{12}+y_{f} \\
d_{F 1}+z_{f} \\
-\sin \left[\phi_{0}\right] \\
0 \\
-\cos \left[\phi_{0}\right]
\end{array}\right] \text {, }} \\
& {\left[\begin{array}{l}
x_{4} \\
y_{4} \\
z_{4} \\
a_{4} \\
b_{4} \\
c_{4}
\end{array}\right]=\left[\begin{array}{c}
d_{23}-\sin \left[\phi_{0}\right] d_{34}+x_{f} \\
-d_{12}+y_{f} \\
-\cos \left[\phi_{0}\right] d_{34}+d_{F 1}+z_{f} \\
\sin \left[\theta_{0}\right] \sin \left[\phi_{0}\right] \\
\cos \left[\theta_{0}\right] \\
\cos \left[\phi_{0}\right] \sin \left[\theta_{0}\right]
\end{array}\right],} \\
& {\left[\begin{array}{l}
x_{4} \\
y_{4} \\
z_{4} \\
a_{4} \\
b_{4} \\
c_{4}
\end{array}\right]=\left[\begin{array}{c}
d_{23}-\sin \left[\phi_{0}\right] d_{34}+x_{f} \\
-d_{12}+y_{f} \\
-\cos \left[\phi_{0}\right] d_{34}+d_{F 1}+z_{f} \\
\sin \left[\theta_{0}\right] \sin \left[\phi_{0}\right] \\
\cos \left[\theta_{0}\right] \\
\cos \left[\phi_{0}\right] \sin \left[\theta_{0}\right]
\end{array}\right]} \\
& =\left[\begin{array}{c}
-r_{\theta_{0}} \sin \left[\theta_{0}\right] \sin \left[\phi_{0}\right] \\
-r_{\theta_{0}} \cos \left[\theta_{0}\right] \\
-r_{\theta_{0}} \sin \left[\theta_{0}\right] \cos \left[\phi_{0}\right] \\
\sin \left[\theta_{0}\right] \sin \left[\phi_{0}\right] \\
\cos \left[\theta_{0}\right] \\
\cos \left[\phi_{0}\right] \sin \left[\theta_{0}\right]
\end{array}\right]
\end{aligned}
$$




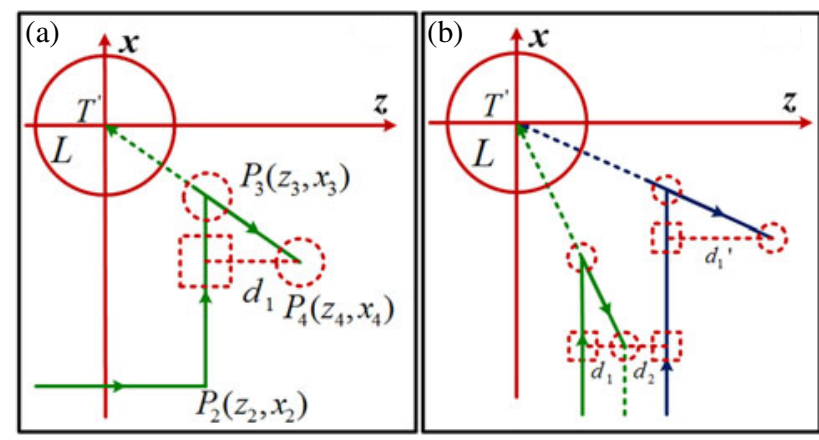

Figure 8. Feasible region of $P_{3}$ : (a) single light (b) two lights.

$$
\left\{\begin{array}{l}
d_{23}=x_{4}-x_{f}+\sin \left[\phi_{0}\right]\left(z_{f}+d_{F 1}-z_{4}\right) / \cos \left[\phi_{0}\right] \\
d_{12}=y_{f}-y_{4} \\
d_{34}=\left(z_{f}+d_{F 1}-z_{4}\right) / \cos \left[\phi_{0}\right] \\
L P L_{\text {total }}=d_{F 1}\left(1+\sec \left[\phi_{0}\right]+\tan \left[\phi_{0}\right]\right) \\
\quad+x_{4}-x_{f}-y_{4}+y_{f}+\left(-z_{4}+z_{f}\right) \\
\quad \times\left(\sec \left[\phi_{0}\right]+\tan \left[\phi_{0}\right]\right)+r_{\theta_{0}} .
\end{array}\right.
$$

Equations (2c) and $(2 d)$ show that all distances and positions in Figure 7 can be expressed as a function of $d_{F 1}$, which can be related to the $z$ position of mirror 3. Besides $d_{F 1}$, the total LPL is decided by two parts, namely, the position of the emergence ports and the distribution angles of the incident ports. Again, the same conclusion can be obtained from Section 2.2. Therefore, the correspondence relationship between the emergence and incident ports is the core problem that the algorithm needs to solve. Considering that the LPL varies with $P_{3}$, fixing the position of mirror 3 is also necessary.

\section{Base-line algorithm}

\subsection{Feasible region of $P_{3}$ in the $X Z$ plane}

The multi-beam BGS model has ensured that there will be no intersection among the layers; in order to avoid intersection among beams in the same layer, the position of $P_{3}$ is constrained in a feasible region. Considering the situation in Figure 8(a), in which only one light exists in the layer, the feasible region of $P_{3}$ is confined by the following conditions. First, as discussed above, $P_{3}$ should be in the projection line segment of $P_{4} T^{\prime}$. Second, considering the sizes of the laser beams and mirrors as the red dotted circle and square marked in the figure and supposing that they equal $D$, then the distance between $P_{4}$ and $P_{2} P_{3}, d_{1}$, must be larger than $D$ to ensure that no obstruction exists in the light paths of the laser beams in the same layer. Third, according to the actual physical conditions, the room must be constructed for a diagnostic instrument in the polar areas of the target chamber. Assuming that this room is cylindrical with the radius $L$, all lights and mirrors must be situated outside of this cylinder, that is, the distance between $P_{3}$ and $T^{\prime}$ should be larger than $L+D$. All three conditions are listed in Equation (3a). In this manner, the feasible region of $P_{3}$, in which two lights are in the same layer as shown in Figure 8(b), can be found from Equation (3b),

$$
\begin{aligned}
& \left\{\begin{array}{l}
\left|x_{3}\right|<\left|x_{4}\right|, \quad x_{3}<0, \quad z_{3}=x_{3} \cot \varphi \\
\sqrt{x_{3}^{2}+z_{3}^{2}}=\left|x_{3} / \sin \varphi\right|>L+D \\
\left|z_{3}\right|<\left|z_{4}\right|, \quad z_{3}>0, \quad d_{1}=\left|z_{3}-z_{4}\right|>D
\end{array}\right. \\
& \begin{cases}\left|x_{3}^{\prime}\right|<\left|x_{4}^{\prime}\right|, \quad x_{3}^{\prime}<0 \\
\left|z_{3}^{\prime}\right|<\left|z_{4}^{\prime}\right|, \quad z_{3}^{\prime}>0, \quad d_{1}^{\prime}=\left|z_{3}^{\prime}-z_{4}^{\prime}\right|>D \\
\left|z_{3}^{\prime}\right|>\left|z_{4}\right|, \quad d_{2}=\left|z_{3}^{\prime}-z_{4}\right|>D \\
z_{4}^{\prime}=x_{3}^{\prime} \cot \varphi, \quad\left|x_{3}^{\prime} / \sin \varphi^{\prime}\right|>L+D .\end{cases}
\end{aligned}
$$

\subsection{Base-line algorithm}

We take NIF as an example to interpret the algorithm. The incident ports are distributed in the target chamber at four altitudes, namely, $23.5^{\circ}, 30^{\circ}, 44.5^{\circ}$ and $50^{\circ}{ }^{[15]}$. The clear radius $r$ is properly selected to render the lights incident to the ports at $23.5^{\circ}$ and $30^{\circ}$ in the same layer. The target chamber is divided into four parts, namely, top, bottom, left and right. Then, in each section of the target chamber, the incident ports are distributed in three layers with four ports. In addition, the emergence ports are divided into three groups according to the incident layers, as shown in Figure 9. Consideration of the corresponding relations of the four lights in the same layer is unnecessary. $P 11$ is connected to $B 11, P 12$ to $B 12, P 13$ to $B 13$ and $P 14$ to $B 14$. In the base-line algorithm, the corresponding relations are fixed, whereas the positions of the emergence ports are variables. The core of the base-line algorithm is to adjust the relative positions of emergence ports to ensure equal LPLs among all beam quads. In this way, the correspondence between the emergence and incident ports is transformed to the calculation of the positions of all the emergence ports, which will make construction and calculation of the model much easier.

The detailed process is illustrated in Figure 10. Taking the first layer as an example, a base-line $B L 01$ is defined for this layer, and then the distances between the base-line and the center of each beam quad are designated as $D q 1 i$ ( $i=$ $1,2,3,4)$. The LPLs of the four lights are calculated, and the different $D q 1 i$ are adjusted to ensure the same path length $d 1$ for all of them. By this method, the shared path lengths in the second and third layers will be $d 2$ and $d 3$. What should be noticed is that the shared LPL of the layer varies with the base-line of the layer. By comparing the three shared LPLs, $d 1, d 2$ and $d 3$, and adjusting the base-lines of the respective layers, then the 12 beam quads in the same group will all share the same LPL. Given that $B L 0 j$ and 


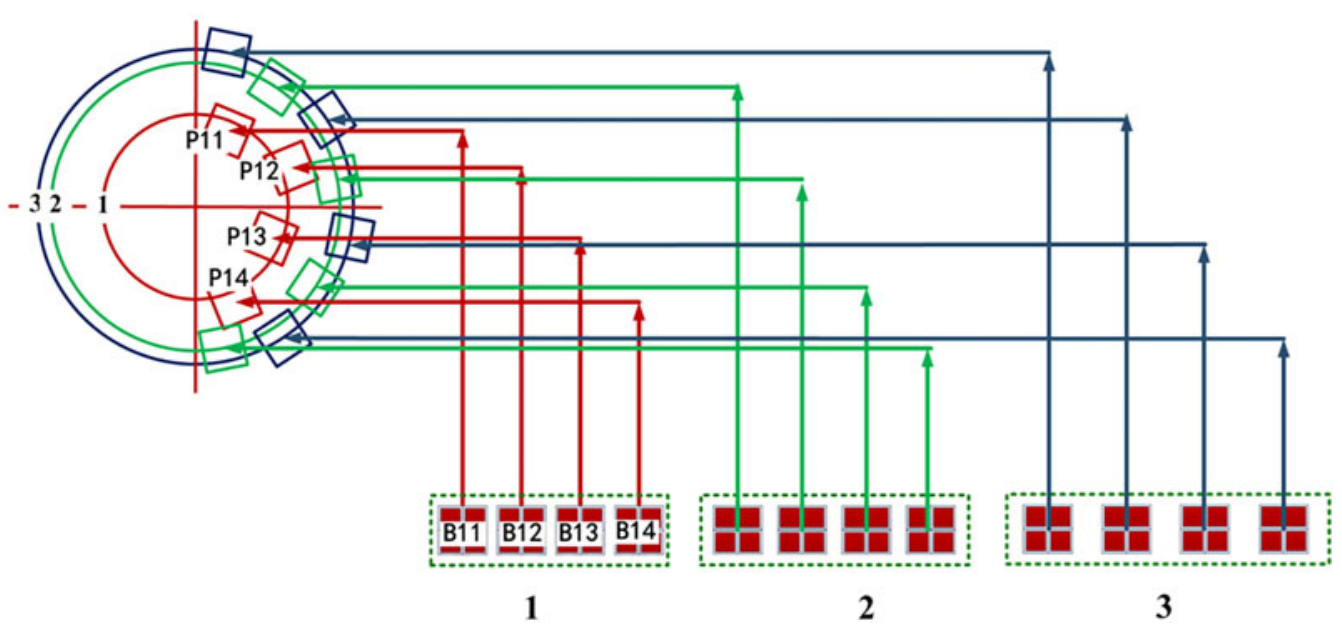

Figure 9. The emergence ports are divided into three groups according to the incident layers.

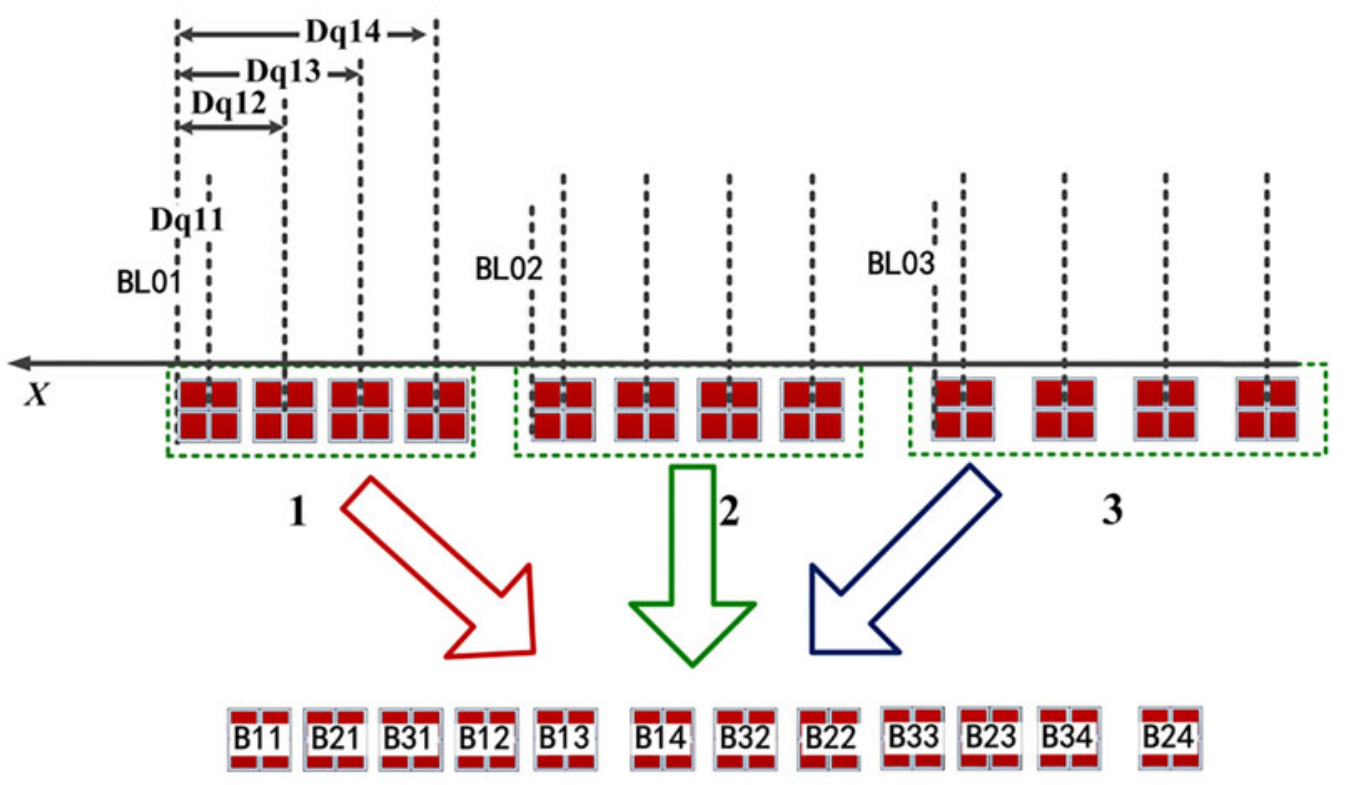

Figure 10. The calculation process of the base-line algorithm.

$\operatorname{Dqji}(j=1,2,3 ; i=1,2,3,4)$ are all fixed, the actual position of the center of the 12 quads can be calculated. Then, the quads can be combined and the distribution of the emergence ports in this part can be obtained.

According to the base-line algorithm, the BGS of a 192beam-line laser driver is arranged. Figure 11 shows the right and bottom parts of the target area, whereas the whole BGS is plotted in Figure 12.

Comparing the calculated BGS arrangement with the NIF BGS, the calculated LPL varies from 57.2 to $63.6 \mathrm{~m}$, which is considerably shorter than that of NIF, which varies from 69.7 to $81.7 \mathrm{~m}^{[10]}$. Considering the radiation shielding that is built in the target area, as shown in Figure 13, the first two mirrors in each beam line must be outside, causing a longer LPL at NIF. Besides the LPL, the BGS arranged through the base-line algorithm is very similar to the real NIF BGS. The base-line algorithm provides the possibility of avoiding heavy calculations of the corresponding relations. Thus, the arrangement of the BGS of a laser driver is simple and efficient.

\section{Boundary conditions and maximum laser beams}

Due to the base-line algorithm, the arrangement of a multibeam laser driver BGS is disregarded. However, discounting this type of BGS does not mean that the number of beam lines can be increased to any expected value to obtain higher laser energy and power. The practical boundary conditions of target area and chamber need to be considered. The 


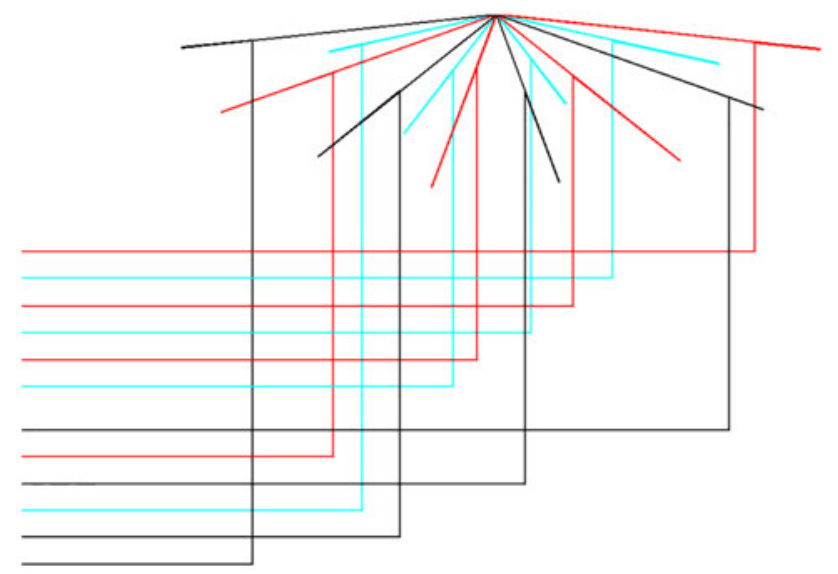

Figure 11. The calculated arrangement of the BGS in the right and bottom parts of the target area.

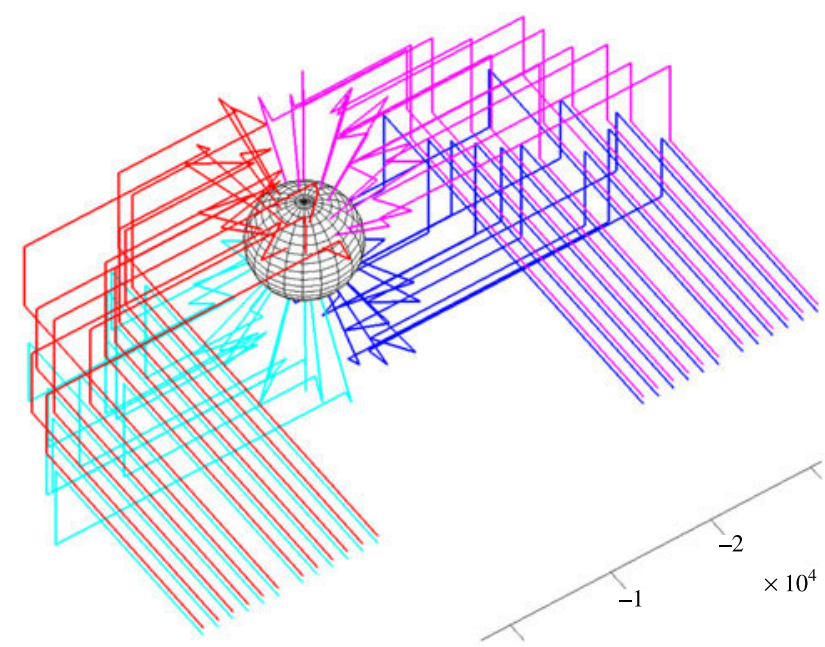

Figure 12. The whole BGS of a 192-beam-line laser driver.

following relative parameters of the real target area involved in BGS arrangement can be generalized and classified, as shown in Figure 14: the radius of the target chamber $R$, the radius of the cylindrical room $L$, the radius of the radiation shielding building $R_{S}$, the distribution of the incident ports $(\theta, \varphi)$, the clear radius $r$, the volume of the target area $X \times Y \times Z$, the starting position of the emergence ports $X_{0}$, the total number of laser beams $N$, the size of a beam quad $D_{q}$ and the distance between beam quads $D_{q q}$.

According to Figure 14, as the number of laser beams increases, all the beams and mirrors must be inside the target area. Considering radiation shielding, the starting position of the emergence ports must be outside the building, and the last two or three mirrors must be inside. In addition, the distance between beam quads must be larger than a beam quad. From the side of the target area, the boundary conditions can be

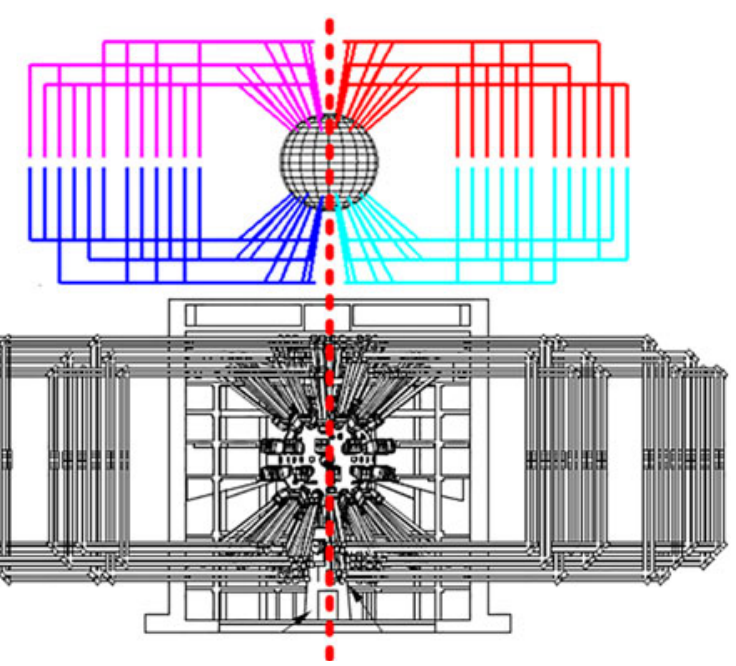

Figure 13. Comparison of the calculated BGS and the NIF BGS.

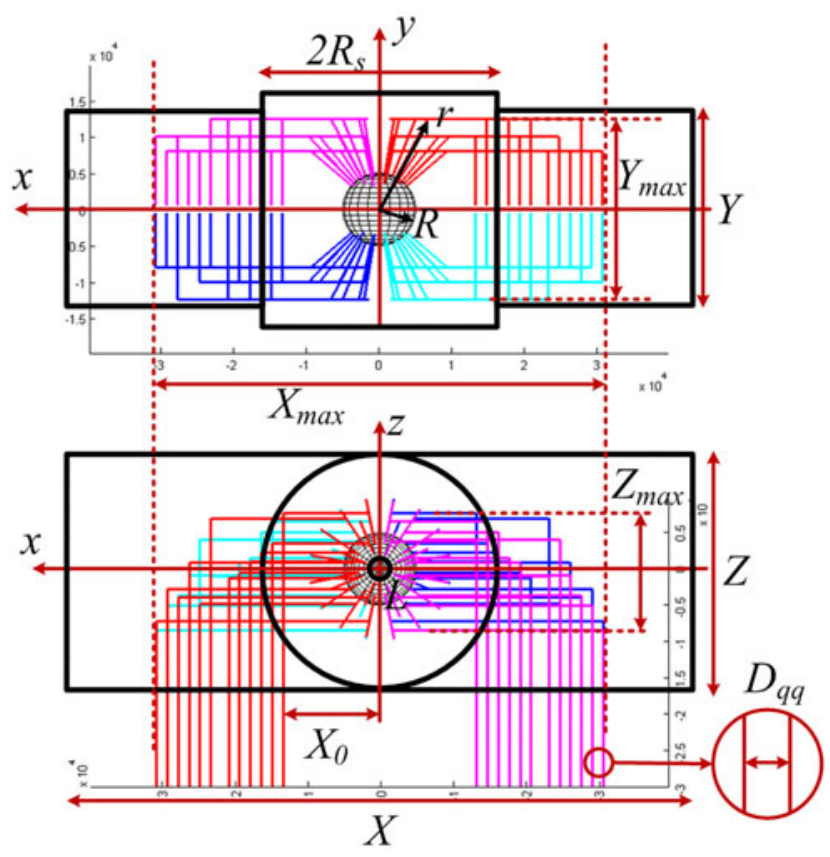

Figure 14. Parameters involved in BGS arrangement.

expressed as shown in Equation (4),

$$
\left\{\begin{array}{l}
X_{\max }=2\left|X_{f \max }\right|<X, \\
Z_{\max }=2 r_{\theta} \sin \theta \cos \varphi<Z, \\
Y_{\max }=2 r_{\theta} \cos \theta<Y, \\
r_{x z \max }=r_{\theta} \sin \theta<R_{S}, \\
X_{0}=\left|X_{f \min }\right|>R_{s}, \\
D_{q q}=1.2-1.5 D_{\text {quad }} .
\end{array}\right.
$$

In Equation (4), $X_{f \max }$ and $X_{f \min }$ are the maximum and minimum values of the emergence port position in the $x$ direction. Still taking the parameters of the NIF target 


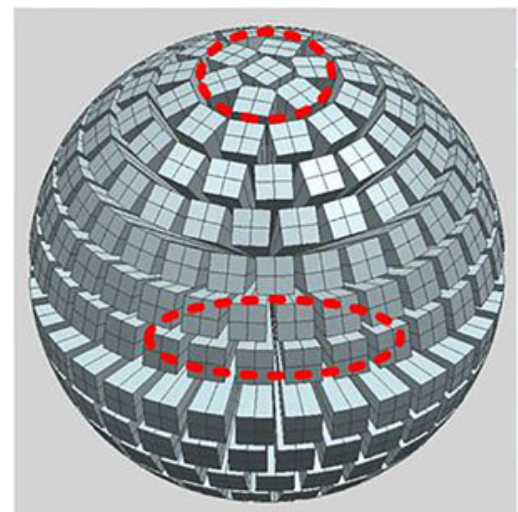

\begin{tabular}{|c|c|}
\hline Degree & Ports \# \\
\hline 0 & 1 \\
\hline 11.5 & 6 \\
23 & 12 \\
34.5 & 17 \\
46 & 22 \\
57.5 & 26 \\
69 & 29 \\
80.5 & 30 \\
\hline
\end{tabular}

Figure 15. Extreme situation: the whole target chamber surface is used for incident light ports.

area $^{[16]}$, the maximum number of laser beams will vary from 266 to 304 based on different values of $D_{q q}$.

From the side of the target chamber, we consider the extreme situation plotted in Figure 15. Considering a 10$\mathrm{m}$-diameter target chamber, if the incident light ports are distributed over the whole chamber surface, we obtain 16 cones of ports with different incident angles and different ports in separate cones, and the total number of laser beams will be 1144 . Given the constraints of indirect drivers, only incident angles that are located between $20^{\circ}$ and $60^{\circ}$ will be suitable ${ }^{[16]}$. Thus, the four cones in the middle latitude will be selected in the upper half-sphere, as shown in the table in Figure 15. In this manner, 154 ports will be suitable. However, considering the mechanic stability of the target chamber, distribution of the ports in the whole cone is impossible. Furthermore, certain ports will be spared for the physical diagnostic equipment. Thus, half of the 154 ports can be used for incident quads, and the maximum number of laser beams that a 10-m-diameter chamber can accommodate is 308 .

According to the boundary conditions of both the target chamber and the target area, and considering that the beam count must be divisible by 48 for a direct-drive cylindrical hohlraum ${ }^{[16]}$, the maximum number of laser beams is 288 for the NIF target chamber and target area.

Based on the discussion above, we can draw several conclusions. A maximum count of laser beams exists for a specific target area and chamber, so increase of the total laser energy by increasing the beam lines is feasible to a certain extent. More laser beams can be obtained by enlarging the target area and chamber. In addition, for 336 laser beams, the chamber will be about $12 \mathrm{~m}$ in diameter. In larger chambers, the focus of the wedged focus lens in the FOA needs to be redesigned to retain the focus. However, with a larger focus, the focus spots in the target will also be enlarged, which may introduce several complicated problems in the target hohlraum ${ }^{[17,18]}$. Apart from enlarging the target area and chamber, which is costly, the development of other drive

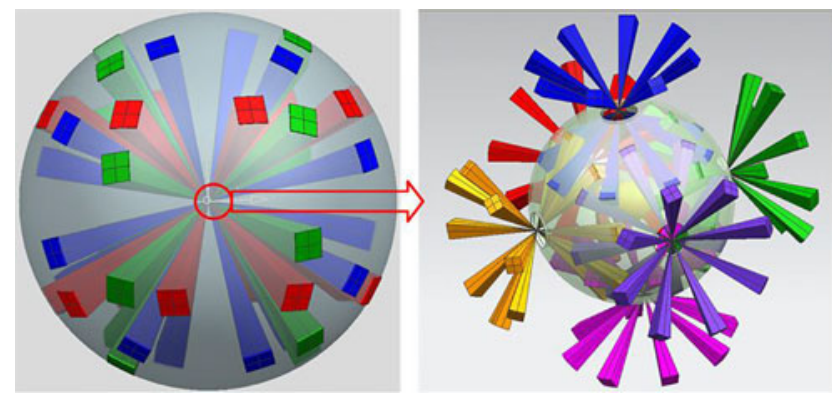

Figure 16. Port distribution in the target chamber and the 6LEH spherical hohlraum.

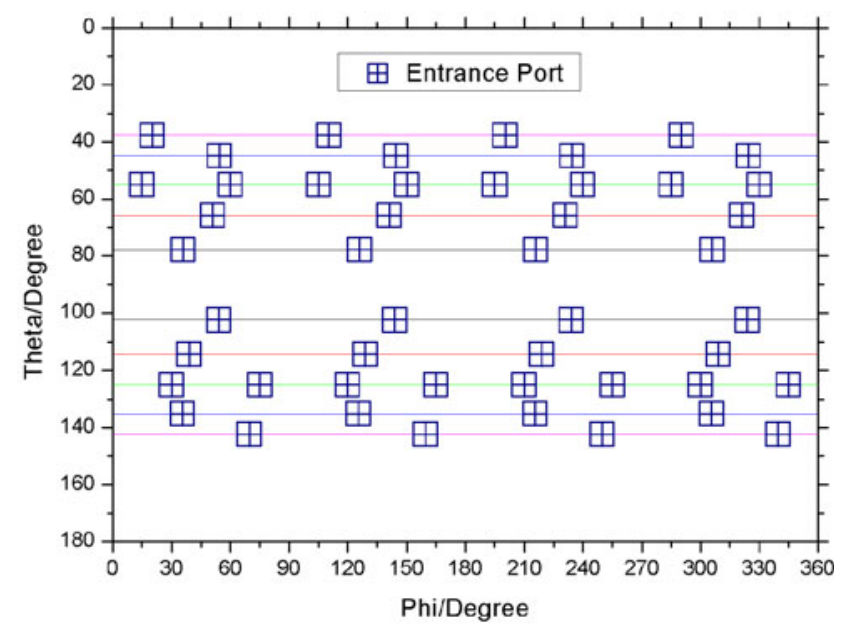

Figure 17. Scheme of the incident port distribution in the target chamber in the theta/phi plane.

targets, such as direct-drive targets ${ }^{[19]}$ and hohlraums with multi-laser entrance holes ${ }^{[20,21]}$, is beneficial.

\section{Six-laser-entrance-hole spherical hohlraum compati- bility}

In 2014, Lan et al. ${ }^{[22]}$ proposed a spherical hohlraum with six laser entrance holes that possess high symmetry and low backscatter. According to the laser arrangement and constraints of the octahedral hohlraum, the incident port distribution in the target chamber for 192 laser beams and the lasers entering the six-laser-entrance-hole (6LEH) hohlraum are plotted in Figure 16. In addition, the incident port distribution can be calculated and plotted in the theta/phi plane of the target chamber, as shown in Figure 17. From Figure 17, it can be seen that the distribution is very similar to that of an indirect-drive cylindrical hohlraum and the ports are situated at different altitudes with different longitudes. by placing both port distributions in the same reference system, the expected results can be obtained. As seen in Figure 18, most of the 6LEH ports are found near the existing NIF 


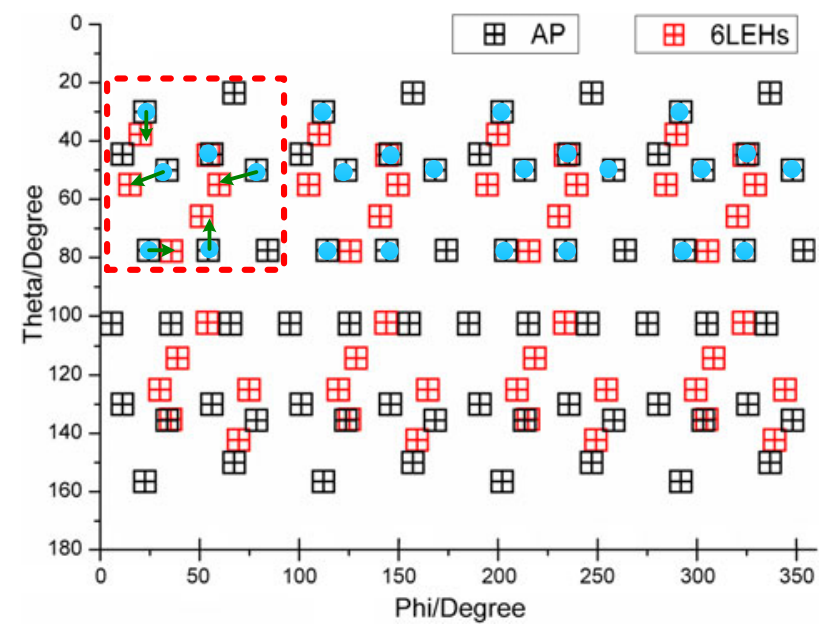

Figure 18. NIF indirect/direct drive and 6LEH incident ports in the theta/phi plane. AP means all ports, which includes direct and indirect ports. Ports with dots indicate that the FOA needs to move to transform.

ports $^{[23]}$, whereas several are coincident, which means that these ports in the NIF chamber can be directly used for a 6LEH hohlraum. For most of the 6LEH ports, if the FOAs of neighboring ports are rotated, as represented by the dots in Figure 18, by small angles while maintaining the aim toward the center of the target chamber, the incident angles of the 6LEH hohlraum can be met easily. Figure 19 is one eighth of the port distribution of Figure 18, indicated by the red dotted rectangle marked in Figure 18. As the figure shows that the longest rotation curve in the target chamber is smaller than the size of two beam quads, a larger hole, probably twice the size of the origin one, can be opened in the dotted ports to accommodate the rotation of the FOA and realize the switch between these two different types of targets. The rotation direction should be along the latitude or longitude so that the movement of the mirrors in the BGS will be constrained in the $x$ and $z$ directions and the change of the BGS will be as small and easy as possible.

\section{Conclusions}

In summary, a BGS model is constructed, and a baseline algorithm is proposed to solve the BGS arrangement in multi-beam laser drivers. According to the boundary conditions, the maximum number of laser beams to obtain higher laser powers and energies for NIF is 288. In addition, more laser beams require a larger chamber, which will introduce an undefined factor to ignition. Through rotation of the FOAs, the target chamber becomes highly compatible with the 6LEH spherical hohlraum. BGS transformation is also relatively easy to achieve. However, considerable work still needs to be accomplished to design a moveable FOA and the detailed steps of switching the BGS between two different types of targets.

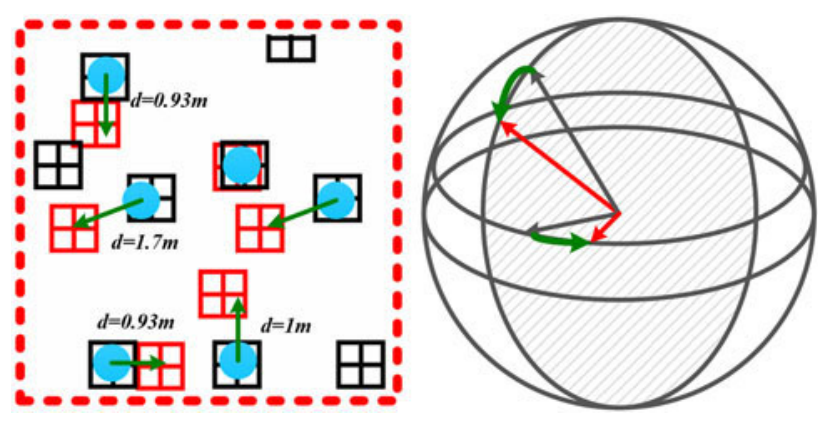

Figure 19. The FOAs are rotated by small angles while maintaining the aim towards the center of the target chamber.

\section{Acknowledgements}

The authors would like to acknowledge Dr. B. Wang, Dr. K. You, and Dr. X. Jiao for their helpful discussion and assistance in BGS code writing and model drawing.

\section{References}

1. E. I. Moses, Nucl. Fusion 49, 104022 (2009).

2. S. E. Bodner, A. J. Schmitt, and J. D. Sethian, High Power Laser Sci. Eng. 1, 2 (2013).

3. E. M. Campbell, in Proceedings of International Conference on Plasma Physics and Controlled Nuclear Fusion Research CONF-9210315-2 (1992).

4. J. L. Kline, D. A. Callahan, S. H. Glenzer, N. B. Meezan, J. D. Moody, D. E. Hinkel, O. S. Jones, A. J. MacKinnon, R. Bennedetti, R. L. Berger, D. Bradley, E. L. Dewald, I. Bass, C. Bennett, M. Bowers, G. Brunton, J. Bude, S. Burkhart, A. Condor, J. M. Di Nicola, P. Di Nicola, S. N. Dixit, T. Doeppner, E. G. Dzenitis, G. Erbert, J. Folta, G. Grim, S. Glenn, A. Hamza, S. W. Haan, J. Heebner, M. Henesian, M. Hermann, D. G. Hicks, W. W. Hsing, N. Izumi, K. Jancaitis, O. S. Jones, D. Kalantar, S. F. Khan, R. Kirkwood, G. A. Kyrala, K. LaFortune, O. L. Landen, L. Lagin, D. Larson, S. L. Pape, T. Ma, A. G. MacPhee, P. A. Michel, P. Miller, M. Montincelli, A. S. Moore, A. Nikroo, M. Nostrand, R. E. Olson, A. Pak, H. S. Park, J. P. Patel, L. Pelz, J. Ralph, S. P. Regan, H. F. Robey, M. D. Rosen, J. S. Ross, M. B. Schneider, M. Shaw, V. A. Smalyuk, D. J. Strozzi, T. Suratwala, L. J. Suter, R. Tommasini, R. P. J. Town, B. Van Wonterghem, P. Wegner, K. Widmann, C. Widmayer, H. Wilkens, E. A. Williams, M. J. Edwards, B. A. Remington, B. J. MacGowan, J. D. Kilkenny, J. D. Lindl, L. J. Atherton, S. H. Batha, and E. Moses, Phys. Plasmas 20, 056314 (2013).

5. C. A. Haynam, R. A. Sacks, P. J. Wegner, M. W. Bowers, S. N. Dixit, G. V. Erbert, G. M. Heestand, M. A. Henesian, M. R. Hermann, K. S. Jancaitis, K. R. Manes, C. D. Marshall, N. C. Mehta, J. Menapace, M. C. Nostrand, C. D. Orth, M. J. Shaw, S. B. Sutton, W. H. Williams, C. C. Widmayer, R. K. White, S. T. Yang, and B. M. V. Wonterghem, J. Phys.: Conf. Ser. 112, 032004 (2008).

6. J. A. Glaze and R. O. Godwin, in Proceedings of Topical Meeting on Inertial Confinement Fusion WE1/1 (1977).

7. M. Rabeau, J. H. Pitts, J. F. Mengue, and G. Maurin, in Proceedings of 14th IEEE/Npss Symposium: Fusion Engineering 1175 (1992). 
8. M. Rabeau, J. H. Pitts, J. F. Mengue, and G. Maurin, Fusion Technol. 23, 337 (1993).

9. R. E. English, C. W. Laumann, J. L. Miller, and L. G. Seppala, in Proceedings of International Optical Design Conference 1998726 (1998).

10. J. L. Miller, R. E. English, R. J. Korniski, and J. M. Rodgers, in Proceedings of Third International Conference on Solid State Lasers for Application to Inertial Confinement Fusion 294 (1999).

11. N. Fleurot, C. Cavailler, and J. L. Bourgade, Fusion Eng. Des. 74, 147 (2005).

12. M. L. Andre, Fusion Eng. Des. 44, 43 (1999).

13. W. Zheng, X. Zhang, X. Wei, F. Jing, Z. Sui, K. Zheng, X. Yuan, X. Jiang, J. Su, H. Zhou, M. Li, J. Wang, D. Hu, S. He, Y. Xiang, Z. Peng, B. Feng, L. Guo, X. Li, Q. Zhu, H. Yu, Y. You, D. Fan, and W. Zhang, J. Phys.: Conf. Ser. 112, 032009 (2008).

14. M.-Z. Zhu, M.-C. Wang, X.-J. Chen, W.-K. Wu, and G. Chen, Opt. Precis. Eng. 21, 701 (2013).

15. P. Bell, P. Di Nicola, G. P. Grim, D. Kalantar, T. McCarville, J. Klingmann, S. Alvarez, R. Lowe-Webb, J. Lawson, P. Datte, P. Danforth, M. Schneider, J. M. Di Nicola, J. Jackson, C. Orth, S. Azevedo, R. Tommasini, A. Manuel, and R. Wallace, Proc. SPIE 8505, 85050B (2012).
16. U.S. Lawrence Livermore Natl Lab, Inertial Confinement Fusion Annual Report (1997).

17. J. D. Lindl, P. Amendt, R. L. Berger, S. G. Glendinning, S. H. Glenzer, S. W. Haan, R. L. Kauffman, O. L. Landen, and L. J. Suter, Phys. Plasmas 11, 339 (2004).

18. Z. Jiao, Y. Zhang, J. Zhang, and J. Zhu, High Power Laser Sci. Eng. 1, 88 (2013).

19. D. Eimerl, J. Rothenberg, M. Key, S. Weber, C. Verdon, S. Skupsky, J. Soures, and S. Craxton, in Proceedings of 1st Annual International Conference on Solid State Lasers for Application to Inertial Confinement Fusion 170 (1995).

20. D. W. Phillion and S. M. Pollaine, Phys. Plasmas 1, 2963 (1994).

21. J. M. Wallace, T. J. Murphy, N. D. Delamater, K. A. Klare, J. A. Oertel, G. R. Magelssen, E. L. Lindman, A. A. Hauer, P. Gobby, J. D. Schnittman, R. S. Craxton, W. Seka, R. Kremens, D. Bradley, S. M. Pollaine, R. E. Turner, O. L. Landen, D. Drake, and J. J. MacFarlane, Phys. Rev. Lett. 82, 3807 (1999).

22. K. Lan, J. Liu, D. Lai, W. Zheng, and X.-T. He, Phys. Plasmas 21, 010704 (2014).

23. U.S. Lawrence Livermore Natl Lab, National Ignition Facility User Guide (2012). 